\title{
RF Impedance Measurement Calibration
}

\author{
P.J. Matthews and J.J. Song \\ Advanced Photon Source \\ Argonne National Laboratory \\ Argonne, Illinois 60439
}

February 12, 1993

\section{INTRODUCTION}

The coupling impedance of the APS-SR vacuum chamber components has been measured with the coaxial wire method using a synthetic pulse technique [1]. As depicted in figure 1, a vector automatic network analyzer (VANA) such as the HP $8510 \mathrm{~B}$ was used to measure the two-port S-parameters of the device-under-test (DUT). The measured parameters must be corrected to account for the imperfections of the various measurement circuit components such as connectors, cables, etc. The HP $8510 \mathrm{~B}$ contains built-in calibration routines for such corrections [2]. Frequently applied ones include the 1-PORT calibration for reflection only, the THRU \& ISOL calibrations for transmission and coupling measurements and a FULL 2-PORT calibration for 2-port S-parameter measurements. Whenever a reference chamber is available and the DUT has a coaxial structure, any of the above-mentioned calibrations can be used.

In many cases, the DUT is either a resonant circuit (cavity) or a non-coaxial structure. For these devices, calibration using the previously mentioned techniques is difficult due to the lack of a good $50 \Omega$ matching load. Devices for which a $50 \Omega$ load is unavailable can still be calibrated using the TSD or TRL techniques $[3,4]$. The TRL calibration is also a built-in routine on both the HP $8510 \mathrm{~B}$ and the HP $8753 \mathrm{C}$. However, there are certain drawbacks which limit the use of these built-in routines, namely:

1. A limited number of data points in the selected frequency span ( 801 points for the $8510 \mathrm{~B}$ and 1601 points for the $8753 \mathrm{C}$ ).

2. The stop frequency must be a harmonic of the start frequency in the low pass time mode.

3. The limited number of data points coupled with a large frequency span and high $Q$ structures forces one to divide the measurements and calibration into smaller frequency ranges. This requires a separate calibration for each span which reduces repeatability due to the assembly/disassembly of the calibration standards.

Due to these limitations, the built-in routines on the HP $8510 \mathrm{~B}$ are not suitable for many applications of interest including coupling impedance measurements [1]. For these cases, a 


\section{DISCLAIMER}

Portions of this document may be illegible in electronic image products. Images are produced from the best available original document. 


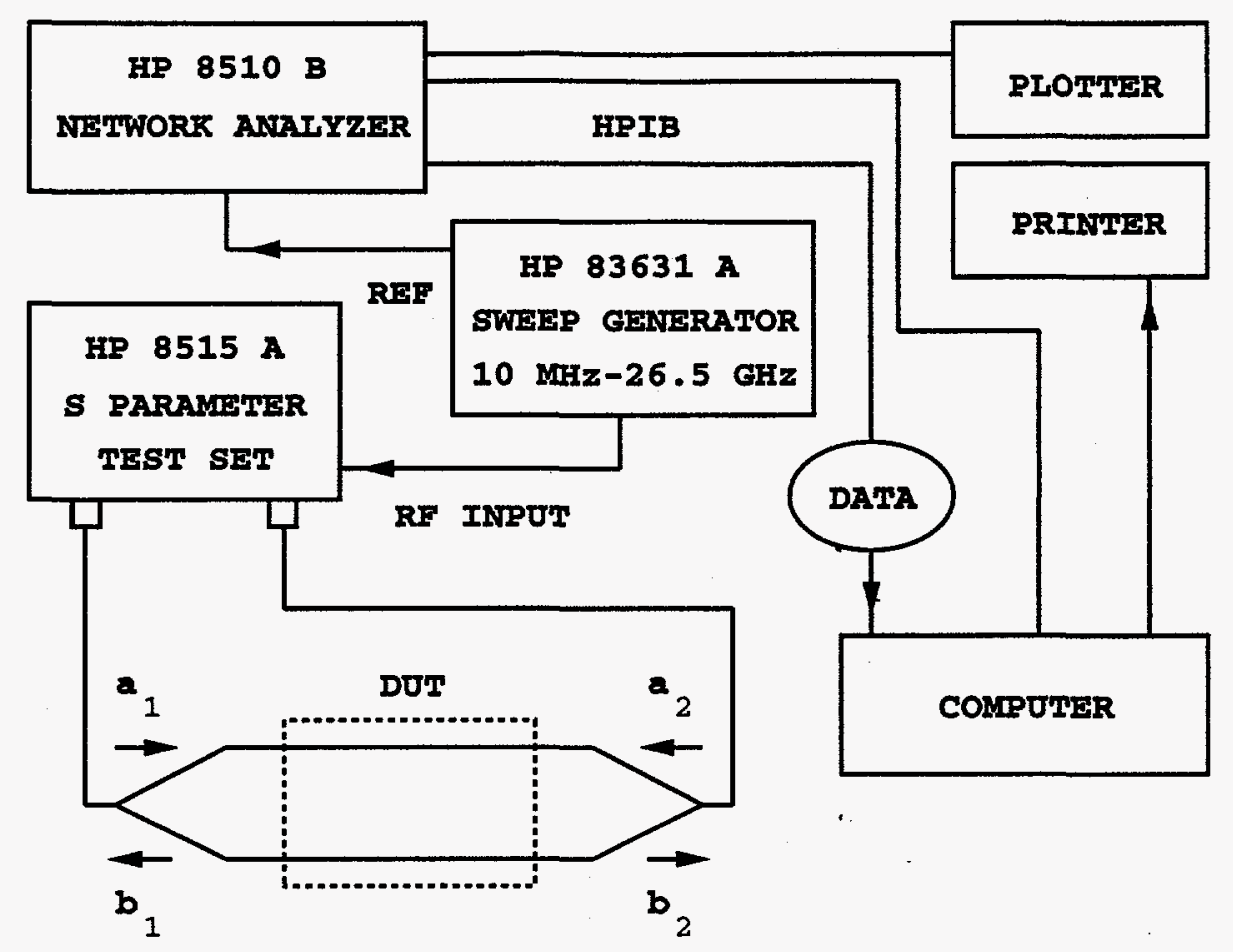

Figure 1: Experimental setup for S-parameter measurement

separate, customized TRL calibration routine should be written. We have chosen to do this using the $\mathrm{C}$ programming language on a personal computer ( $\mathrm{PC})$.

The intent of this note is not to explain all of the available calibration methods in detail. Instead, we will focus on the calibration methods of interest for RF impedance coupling measurements and attempt to explain:

1. the standards and measurements necessary for the various calibration techniques,

2. the advantages and disadvantages of each technique,

3. the mathematical manipulations that need to be applied to the measured standards and devices, and

4. an outline of the steps needed for writing a calibration routine that operates from a remote computer.

For further details of the various techniques presented in this note, the reader should consult the references.

\section{FULL 2-PORT CALIBRATION}

Microwave devices are typically characterized by scattering parameters which relate the incoming and outgoing voltage signals of the various ports. The scattering parameters for a 


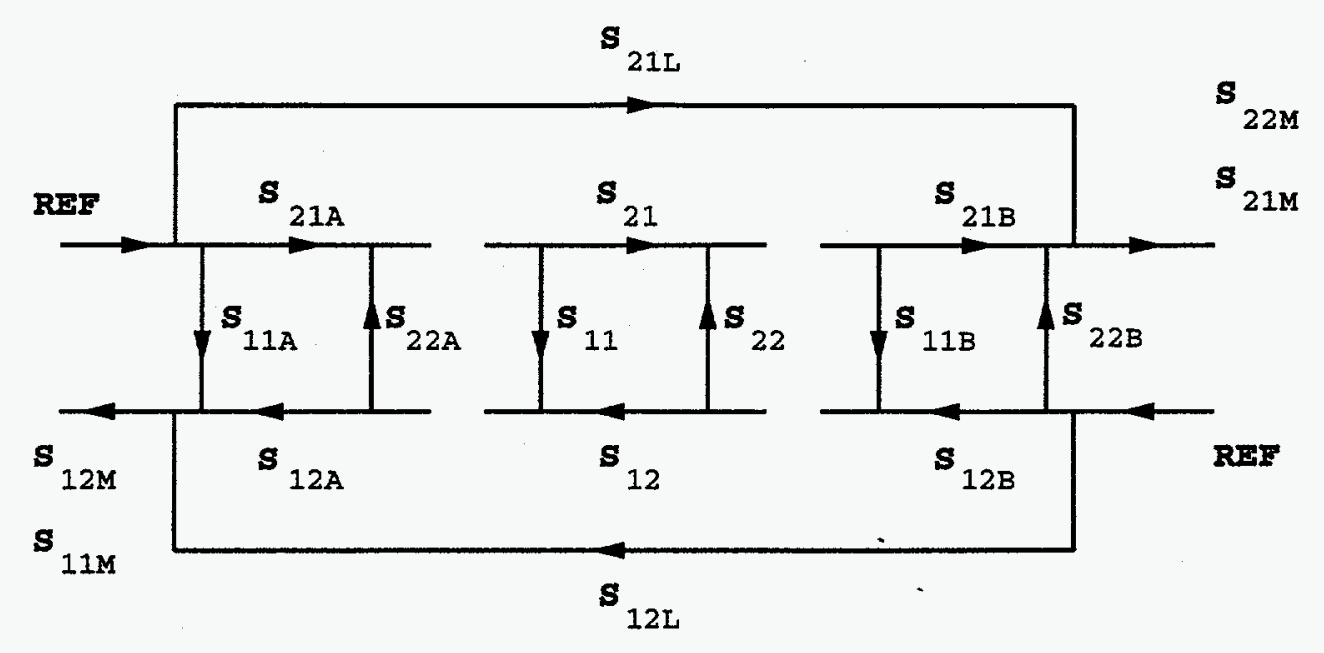

Figure 2: The 10-term error model

two-port device are defined as follows:

$$
\left[\begin{array}{l}
b_{1} \\
b_{2}
\end{array}\right]=\left[\begin{array}{ll}
S_{11} & S_{12} \\
S_{21} & S_{22}
\end{array}\right]\left[\begin{array}{l}
a_{1} \\
a_{2}
\end{array}\right],
$$

where $a_{n}$ and $b_{n}$ are defined by the forward and backward voltages at each port, normalized to the port impedance, $Z_{0 n}$. When discussing calibration techniques, it is oftentimes more convenient to talk in terms of cascadable $\mathrm{T}$-matrices which are defined by

$$
\left[\begin{array}{l}
b_{1} \\
a_{1}
\end{array}\right]=\left[\begin{array}{ll}
T_{11} & T_{12} \\
T_{21} & T_{22}
\end{array}\right]\left[\begin{array}{l}
a_{2} \\
b_{2}
\end{array}\right] .
$$

Simple relationships exist between the terms of the $\mathrm{S}$ and $\mathrm{T}$ matrices.

In general, measurement of the scattering parameters of an unknown device should be corrected for repeatable system errors through a system calibration. Any microwave calibration technique must be capable of representing repeatable system errors and provide a method for correcting uncalibrated measurements. Most methods represent these errors by the scattering response of a hypothetical virtual error network which interfaces the network analyzer to the DUT. The scattering parameters of the virtual error networks are computed from the measurement of known standards. The 'true' parameters of the DUT can then be calculated by stripping the error networks from the measured scattering response of the DUT. The different calibration techniques differ mainly in the proposed error model that is solved and the number and type of standards used. In general, at least three standards are needed for calibration of a two-port device.

The full two-port calibration used in the $8510 \mathrm{~B}$ is based on the work by Vladmir Gelnovatch [5] but uses a more complex 12-term error model. The calibration in [5] which will be described here uses a 10-term error model which includes leakage (see figure 2). The full two-port calibration requires measurements on both ports of the VANA with three standards 
- a known load, an open, and a short. The scattering parameters of a 'through' connection (port one of the VANA connected directly to port two) also need to be measured. The results of these measurements are used to calculate the error vectors $S_{11 A}, S_{22 A}, S_{11 B}, S_{22 B}, S_{21 L}$, $S_{12 L}, S_{12 A} S_{21 A}, S_{12 B} S_{21 B}, S_{22 B} S_{12 A}$, and $S_{21 A} S_{21 B}$ using closed form expressions. Notice that some of the error vectors solved for are actually products of individual error vectors and do not need to be solved for individually. The solution for the embedded scattering parameters of an unknown DUT can then be given in terms of the measured scattering parameters of the DUT and the error vectors by [5]

$$
\begin{aligned}
& S_{11}=\frac{\left(\frac{S_{11 M}-S_{11 A}}{S_{12 A} S_{21 A}}\right)\left[1+S_{11 B}\left(\frac{S_{22 \Lambda}-S_{22 B}}{S_{21 B} S_{12 B}}\right)\right]-S_{11 B}\left(\frac{S_{21 M}-S_{21 L}}{S_{21 B} S_{12 B}}\right)\left(\frac{S_{12 M}-S_{12 L}}{S_{12 A} S_{21 A}}\right)}{D} \\
& S_{22}=\frac{\left(\frac{S_{22 M}-S_{22 B}}{S_{12 B} S_{21 B}}\right)\left[1+S_{22 A}\left(\frac{S_{11 M}-S_{11 A}}{S_{12 A} S_{21 A}}\right)\right]-S_{22 A}\left(\frac{\dot{S_{21 M}}-S_{21 A}}{S_{12 B} S_{21 B}}\right)\left(\frac{S_{12 M N}-S_{12 L}}{S_{12 A} S_{21 A}}\right)}{D}, \\
& S_{21}=\frac{\left(\frac{S_{21 \Lambda \mathcal{L}}-S_{21 L}}{S_{21 B} S_{21 A}}\right)}{D} \\
& S_{12}=\frac{\left(\frac{S_{12 M}-S_{12 L}}{S_{12 A} S_{12 B}}\right)}{D}
\end{aligned}
$$

where $S_{i j M}$ are the measured scattering parameters of the DUT and where

$$
\begin{aligned}
D= & {\left[1+S_{22 A}\left(\frac{S_{11 M}-S_{11 A}}{S_{12 A} S_{21 A}}\right)\right]\left[1+S_{11 B}\left(\frac{S_{22 M}-S_{22 B}}{S_{12 B} S_{21 B}}\right)\right] } \\
& -S_{22 A} S_{11 B}\left(\frac{S_{21 M}-S_{12 L}}{S_{12 A} S_{21 A}}\right)\left(\frac{S_{21 M}-S_{211}}{S_{12 B} S_{21 B}}\right) .
\end{aligned}
$$

The full two-port calibration provides closed form expressions for the error vectors and the de-embedded scattering parameters of the DUT. The measurements and standards needed for calibration are fairly simple. Thus, the full two-port calibration works well for coaxial transmission lines where a good load standard may be fabricated. However, in many cases such as the APS storage ring chamber configuration, the standards required for the full twoport calibration are not practical. In these cases, other calibration techniques become more desirable.

\section{GATING TECHNIQUES}

The time domain 'gating' technique is not a true calibration procedure. However, it does allow one to enhance and perform transmission measurements on localized portions of a DUT. Also it is particularly useful for evaluation of many of the devices to be used in the APS. For these reasons, we will briefly discuss the 'gating' technique.

'Gating' is a time filtering technique which allows one to measure the frequency response at a particular location of the DUT. One way to accomplish this is to transmit a short pulse through the DUT and view the detected signal through a window in the time domain. The window allows one to isolate the pulse which has not undergone a reflection and discard 


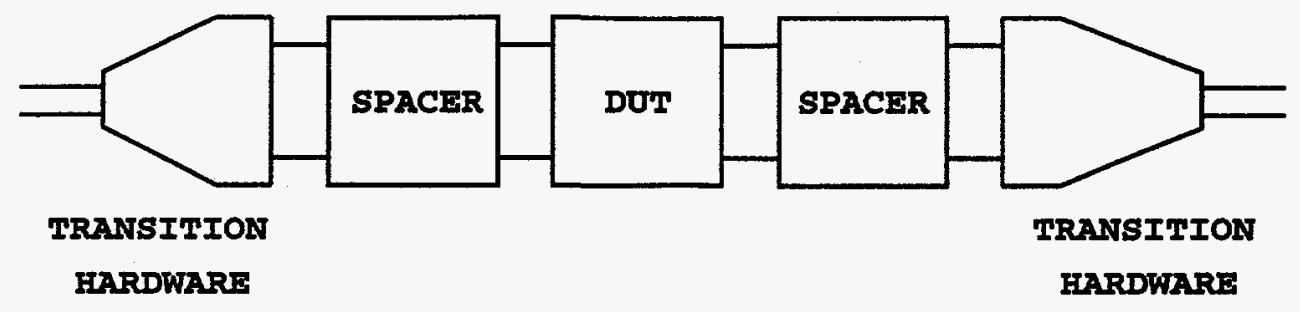

Figure 3: Experimental setup for the time domain gating technique

all other pulses. Another method is to perform the measurement in the frequency domain, convert it to the time domain via an inverse Fast Fourier Transform (FFT), and then perform the windowing. The 'gated' data can then be converted to the frequency domain with a FFT. In this fashion, the frequency response of a particular area of interest of the DUT can be calculated by filtering out the various unwanted reflections.

The basic setup for performing gated time domain measurements is shown in figure 3 . The spacers are needed to ensure that the various pulses from the reflections are well separated and do not overlap in the time domain. The width of the gate must also be considered and depends on the lowest frequency needed as well as the frequency range of interest. To eliminate low frequency overlap, the length of the spacers should be at least $c \tau / 2$ where $\tau$ is the gate width. The low frequency cutoff is given by $\approx 1 / \tau$. It should be noted that the length of the spacers starts to become impractical for frequencies $\approx 100 \mathrm{MHz}$. The gating technique can be better visualized using the signal flow diagram in figure 4 . In this figure, the subscripts $X$ and $Y$ refer to the transition hardware while the spacer sections are represented by the exponential terms. The transmission of this system is given by

$$
S_{21}^{T O T A L}=\frac{S_{21 X} S_{21 Y} S_{21} \mathrm{e}^{\mathrm{i}\left(\theta_{1}+\theta_{2}\right)}}{1-S_{22 X} S_{11} \mathrm{e}^{i 2 \theta_{1}}-S_{11 Y} S_{22} \mathrm{e}^{i 2 \theta_{2}}+S_{22 X} S_{11 Y} \mathrm{e}^{i 2\left(\theta_{1}+\theta_{2}\right)}\left(S_{11} S_{22}-S_{12} S_{21}\right)} .
$$

It is easy to see that by gating around the non-reflected pulse, we can eliminate the reflections in the denominator of equation 8 giving

$$
S_{21}^{G A T E D}=S_{21 X} S_{21 Y} S_{21} \mathrm{e}^{i\left(\theta_{1}+\theta_{2}\right)} .
$$

The time domain gating technique is a powerful measurement technique for transmission measurements. It allows one to filter out the multiple reflections that obscure the desired result. Care must be taken to avoid higher-order mode propagation and aliasing when using the gating technique. Higher-order mode propagation can cause a beating between the different mode pulses giving incorrect results. Aliasing results from using FFT's and again can give erroneous results. Another drawback to the gating technique is the need for short pulses for good spatial resolution.

\section{TRL CALIBRATION}

The drawbacks to the full two-port and the gating techniques point out the need for a direct calibration using simple, realizable standards which are applicable to the various devices 


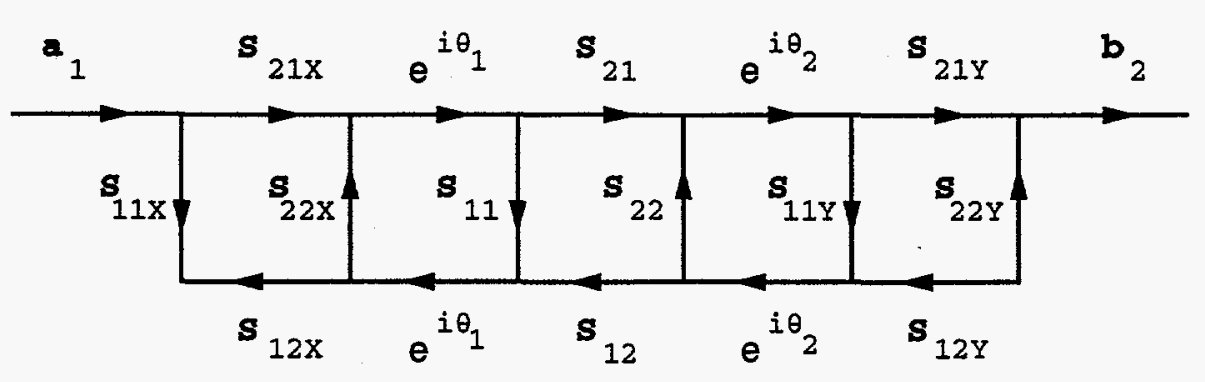

Figure 4: The signal flow diagram for gating

of the APS. The TRL (through, reflect, line) calibration developed by G.F. Engen and C.A. Hoer [4] provides a simple technique for the calibration of devices for which matched loads are difficult to obtain. The method only requires three standards - a through connection, a high reflectivity termination, and a section of uniform line. It should be noted here that the TSD (through, short, delay) calibration [6] is a special case of the TRL calibration where the high reflectivity termination is considered to be a perfect short.

The standards for the TRL calibration are easily realizable even for unusual waveguide configurations. The through standard may contain a short (usually $<1 / 4 \lambda$ ) spacer. Also, it is not necessary to know the reflection coefficient of the short. This value is actually determined during the calibration process. The precise electrical length of the line standard does not need to be known and is also determined during the calibration. The only critical parameter is the impedance, $Z_{0}$, of the delay line.

The basic TRL calibration outlined in [4] is based upon an 8-term error model (see figure 5). The procedure then consists of finding the S-parameters for two two-port virtual error matrices. This calibration therefore ignores the leakage terms, $S_{12 L}$ and $S_{21 L}$, present in the 10-term error model (see figure 2). However, adding in the leakage terms is not difficult and will be discussed later. A more detailed derivation of the TRL calibration equations is given in Appendix A. From figure 5 it is easy to see that the measurement of the uncalibrated $\mathrm{S}$-parameters of a DUT can be written in terms of T-matrices as

$$
T_{M}=T_{A} T_{D U T} T_{B},
$$

where $T_{M}$ represents the measured T-matrix, $T_{D U T}$ represents tha actual T-matrix of the DUT and $T_{A}$ and $T_{B}$ are the virtual error box T-matrices. Once the calibration has been completed and the terms of the virtual error matrices determined, de-embedding the DUT simply consists of stripping off the error matrices

$$
T_{D U T}=T_{A}^{-1} T_{M} T_{B}^{-1} .
$$

This results in explicit expressions for the de-embedded S-parameters. These parameters may be calculated from equation 11 and are given in Appendix B.

The calibration procedure consists of measuring the scattering matrices for the three standards which gives three known scattering matrices, $S_{T}, S_{R}$, and $S_{D}$, containing 10 terms 


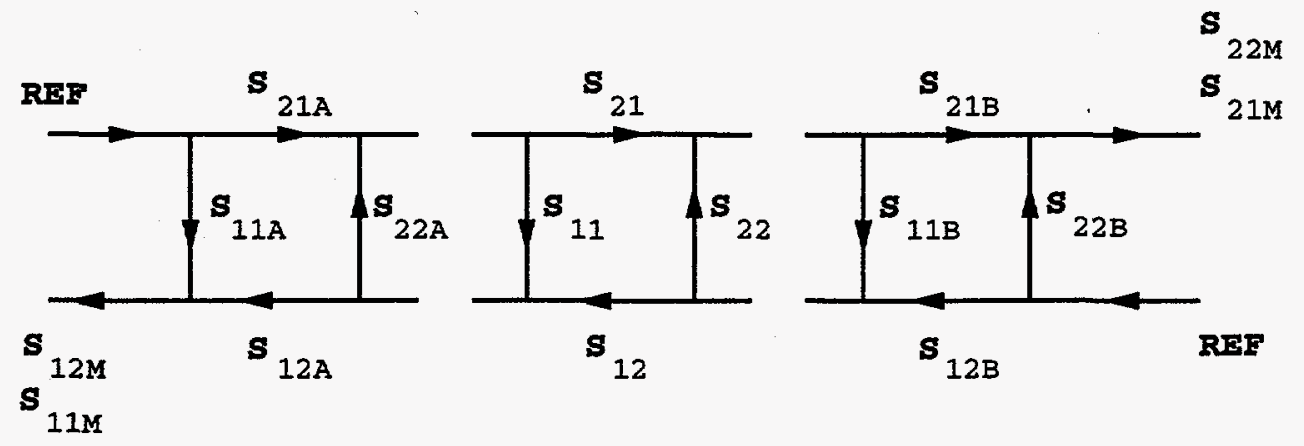

Figure 5: The TRL error model

( $S_{12 R}$ and $S_{21 R}$ are set equal to zero). Given these measurements, we need to find the eight terms of the two error matrices, $S_{A}$ and $S_{B}$. We begin by converting all of the measured S-matrices, $S_{T}, S_{R}$, and $S_{D}$, into T-matrices, $T_{T}, T_{R}$, and $T_{D}$. The T-matrix for the through connection can be represented by

$$
T_{T}=T_{A} T_{B}
$$

while the T-matrix for the line connection can be represented by

$$
T_{D}=T_{A} T_{L} T_{B}
$$

where $T_{L}$ is the $T$-matrix for a uniform line section and is given by

$$
T_{L}=\left[\begin{array}{cc}
\mathrm{e}^{-\gamma l} & 0 \\
0 & \mathrm{e}^{\gamma l}
\end{array}\right]
$$

Solving equation 12 for $T_{B}$ and substituting into equation 13 gives

$$
T_{D} T_{T}^{-1} T_{A}=T_{A} T_{L}
$$

Equation 15 consists of four equations with five unknowns. However, there are only three independent solutions to equation 15 . These solutions give $\left(\frac{t_{11 A}}{t_{21 A}}\right),\left(\frac{t_{12 A}}{t_{22 A}}\right)$, and $\mathrm{e}^{2 \gamma l}$. We have effectively solved for two terms of the $A$ error matrix as well as the electrical length of the delay line. Using this information, we can now solve equation 12 for $T_{B}$ to give $\left(\frac{t_{21 B}}{t_{22 B}}\right)$ and $\left(\frac{t_{12 B}}{t_{11 B}}\right)$ in terms of $\left(\frac{t_{11 A}}{t_{11 A}}\right),\left(\frac{t_{12 A}}{t_{22} A}\right)$ and the measured quantities of $T_{T}$.

We must now make use of the reflection standard. The reflection coefficient measured at port $1, S_{11 R}$, can be written as a function of the reflection coefficient of the termination, $\Gamma_{L}$, and the S-parameters of error-box A

$$
S_{11 R}=S_{11 A}+\frac{S_{12 A} S_{21 A} \Gamma_{L}}{1-S_{22 A} \Gamma_{L}} .
$$

Similarly, we can write for the measured reflection coefficient at the B error box

$$
S_{22 R}=S_{22 B}+\frac{S_{12 B} S_{21 B} \Gamma_{L}}{1-S_{11 B} \Gamma_{L}} .
$$


Equations 16,17 , and 12 can be solved for $\left(\frac{t_{11 A}}{t_{22 A}}\right),\left(\frac{t_{11 B}}{t_{22 B}}\right)$, and $\Gamma_{L}$. At this point the TRL calibration is essentially completed. The S-parameters of the virtual error matrices are given in terms of the solved quantities by

$$
\begin{gathered}
S_{11 A}=\frac{t_{12 A}}{t_{22 A}}, \\
S_{22 A}=-\frac{t_{21 A}}{t_{22 A}}=-\left(\frac{t_{21 A}}{t_{11 A}}\right)\left(\frac{t_{11 A}}{t_{22 A}}\right), \\
S_{12 A} S_{21 A}=\frac{1}{t_{22 A}^{2}}\left(t_{11 A} t_{22 A}-t_{12 A} t_{21 A}\right) \\
=\frac{t_{11 A}}{t_{22 A}}\left\{1-\left(\frac{t_{12 A}}{t_{22 A}}\right)\left(\frac{t_{21 A}}{t_{11 A}}\right)\right\}, \\
S_{11 B}=\frac{t_{12 T}-t_{22 T}\left(\frac{t_{21 A}}{t_{11 A}}\right)\left(\frac{t_{11 A}}{t_{22}}\right)}{t_{22 T}\left(\frac{t_{11 A}}{t_{22 A}}\right)-t_{12 T}\left(\frac{t_{12 A}}{t_{22 A}}\right)}, \\
S_{22 B}=\frac{t_{21 T}\left(\frac{t_{11 A}}{t_{22 A}}\right)-t_{11 T}\left(\frac{t_{12 A}}{t_{22 A}}\right)}{t_{22 T}\left(\frac{t_{11 A}}{t_{22 A}}\right)-t_{12 T}\left(\frac{t_{22 A}}{t_{22 A}}\right)}, \text { and } \\
S_{12 B} S_{21 B}=\frac{t_{11 B}}{t_{22 B}}+S_{11 B} S_{22 B} \\
=\frac{t_{11 T}-t_{21 T}\left(\frac{t_{22 A}}{t_{11 A}}\right)\left(\frac{t_{11 A}}{t_{22 A}}\right)}{t_{22 T}\left(\frac{t_{11 A}}{t_{22 A}}\right)-t_{12 T}\left(\frac{t_{12 A}}{t_{22 A}}\right)}+S_{11 B} S_{22 B} .
\end{gathered}
$$

As is evident from the above equations, the TRL calibration solves only for the error matrix product, $S_{12} S_{21}$. It is impossible, on physical grounds, to separate these two S-parameters. We have thus solved for only six of the eight terms contained in the two virtual error boxes. By hypothesizing that the two error matrices share equally in any apparent nonreciprocity, we can write

$$
S_{12 A}= \pm \sqrt{ \pm S_{12 A} S_{21 A} \sqrt{\frac{S_{12 T}}{S_{21 T}}}}
$$

and

$$
S_{21 A}=\frac{S_{12 A} S_{21 A}}{S_{12 A}} .
$$

Similar equations exist for the second error matrix by substituting $B$ for $A$ in the above equations. All eight terms of the two error matrices are now known and the calibration is complete.

As was mentioned before, the TRL calibration does not contain the two leakage terms of the 10-term error model (see figure 2). The leakage terms are usually negligible but are easily included in the TRL calibration. This is accomplished by placing terminations at the calibration plane for which $S_{12}=S_{21}=0$. The high reflectivity standard of the 
TRL calibration satisfies this condition. By measuring $S_{12}$ and $S_{21}$ with these terminations in place, we obtain the leakage terms $S_{12 L}$ and $S_{21 L}$ respectively (see figure 2). The deembedding procedure is now the same except that we must replace $S_{12 M}$ with $\left(S_{12 M}-S_{12 L}\right)$ and $S_{21 M}$ with $\left(S_{21 M}-S_{21 L}\right)$ in the de-embedding equations given in Appendix B.

The TRL calibration is well suited for impedance measurements of the various APS devices. The standards are simple and easily realizable even for unusual device geometries. The calibration process is straightforward and results in explicit expressions for the error terms. De-embedding is also performed using explicit expressions. Additionally, the TRL calibration process can be easily implemented on a remote computer to overcome the limitations of the built-in routines on the HP $8510 \mathrm{~B}$ and $8753 \mathrm{C}$ network analyzers. The TRL calibration technique does possess some limitations. Higher-order modes can affect the calibration and must be eliminated. For best accuracy, the through standard should be less than $1 / 4 \lambda$ at the highest frequency measured and the delay or line standard should be less than $1 / 2 \lambda$ longer at the highest frequency. However, the difference in length between these standards should be discernible (greater than $\approx 20^{\circ}$ ) at the lowest frequency. These restrictions limit the frequency span for a given set of standards to an 8:1 ratio. Thus, for a larger desired frequency range, multiple standards and calibrations must be applied.

\section{REFERENCES}

[1] J. Song. Experimental study of coupling impedance. Part I: Longitudinal impedance measurement techniques. LS-201, October 22, 1991.

[2] HP Product Note 8510-5A. Network analysis.

[3] R. A. Speciale. A generalization of the TSD network-analyzer calibration procedure, covering $\mathrm{n}$-port scattering parameter measurements, affected by leakage errors. IEEE Transactions on Microwave Theory and Techniques, MTT-25:1100-1115, 1977.

[4] G. F. Engen and C. A. Hoer. "Thru-Reflect-Line": An improved technique for calibrating the dual six-port automatic network analyzer. IEEE Transactions on Microwave Theory and Techniques, MTT-27:987-993, 1979.

[5] V. G. Gelnovatch. A computer program for the direct calibration of two-port reflectometers for automated microwave measurements. IEEE Transactions on Microwave Theory and Techniques, MTT-24:45-47, 1976.

[6] N. R. Franzen and R. A. Speciale. A new procedure for system calibration and error removal in automated s-parameter measurements. In Proc. $5^{\text {th }}$ European Microwave Conf. Hamburg, Germany, Sept. 1-4, 1975. 


\section{Appendix A}

Derivation of the TRL calibration

This derivation follows that given in [4]. Measurement of the scattering matrices for the three standards (through, reflect, line) gives three known scattering matrices, $S_{T}, S_{R}$ and $S_{D}$, containing 10 terms ( $S_{12 R}$ and $S_{21 R}$ are set equal to zero). Given these measurements, we need to find the eight terms of the two error matrices, $S_{A}$ and $S_{B}$ (see figure 5). We begin by converting all of the measured S-matrices, $S_{T}, S_{R}$ and $S_{D}$, into T-matrices, $T_{T}, T_{R}$ and $T_{D}$. The T-matrix for the through connection can be represented by

$$
T_{T}=T_{A} T_{B}
$$

while the T-matrix for the line connection can be represented by

$$
T_{D}=T_{A} T_{L} T_{B},
$$

where $T_{L}$ is the $\mathrm{T}$-matrix for a uniform line section and is given by

$$
T_{L}=\left[\begin{array}{cc}
\mathrm{e}^{-\gamma l} & 0 \\
0 & \mathrm{e}^{\gamma l}
\end{array}\right] \text {. }
$$

Solving equation 26 for $T_{B}$ and substituting into equation 27 gives

$$
T_{D} T_{T}^{-1} T_{A}=T_{A} T_{L}
$$

after some manipulation. The quantity $T_{D} T_{T}^{-1}$ is a measured, known quantity and will be renamed $T_{D T}$ for convenience. We can now write out equation 29 explicitly

$$
\begin{gathered}
t_{11 D T} t_{11 A}+t_{12 D T} t_{21 A}=t_{11 A} \mathrm{e}^{-\gamma l}, \\
t_{11 D T} t_{12 A}+t_{12 D T} t_{22 A}=t_{12 A} \mathrm{e}^{\gamma l}, \\
t_{21 D T} t_{11 A}+t_{22 D T} t_{21 A}=t_{21 A} \mathrm{e}^{-\gamma l} \\
t_{21 D T} t_{12 A}+t_{22 D T} t_{22 A}=t_{22 A} \mathrm{e}^{\gamma l}
\end{gathered}
$$

where $t_{i j T D}$ are the elements of the matrix $T_{D T}$. We now have a system of four equations with five unknowns (the four elements of the matrix $T_{A}$ and $\gamma l$ ). However, taking the determinant of equation 29 gives

$$
t_{11 D T} t_{22 D T}-t_{12 D T} t_{21 D T}=1,
$$

which shows that there are only three independent solutions to equation 29 . We can now solve equations 30 through 33 for the three independent solutions. The ratio of equation 30 to equation 32 and equation 31 to equation 33 gives

$$
t_{21 D T}\left(\frac{t_{11 A}}{t_{21 A}}\right)+\left(t_{22 D T}-t_{11 D T}\right)\left(\frac{t_{11 A}}{t_{21 A}}\right)-t_{12 D T}=0
$$




$$
t_{21 D T}\left(\frac{t_{12 A}}{t_{22 A}}\right)+\left(t_{22 D T}-t_{11 D T}\right)\left(\frac{t_{12 A}}{t_{22 A}}\right)-t_{12 D T}=0 .
$$

Therefore, the ratios $\left(\frac{t_{11 A}}{t_{21 A}}\right)$ and $\left(\frac{t_{12 A}}{t_{22 A}}\right)$ are given by the solutions to the same quadratic equation. The solutions can be written as

$$
\left(\frac{t_{11 A}}{t_{21 A}}\right),\left(\frac{t_{12 A}}{t_{22 A}}\right)=\frac{1}{2 t_{21 D T}}\left\{t_{11 D T}-t_{22 D T} \pm \sqrt{\left(t_{22 D T}-t_{11 D T}\right)^{2}+4 t_{21 D T} t_{12 D T}}\right\} .
$$

It cannot be determined right now which solution corresponds to which ratio. This will be discussed later. We can now take the ratio of equation 33 to equation 32 to arrive at the third solution

$$
\mathrm{e}^{2 \gamma l}=\frac{t_{21 D T}\left(\frac{t_{12 A}}{t_{22 A}}\right)+t_{22 D T}}{t_{12 D T}\left(\frac{t_{21 A}}{t_{11 A}}\right)+t_{11 D T}}
$$

This is all the information that we can get out of equations 30 through 33 . We now know three parameters, $\left(\frac{t_{11 A}}{t_{21 A}}\right),\left(\frac{t_{12 A}}{t_{22 A}}\right)$ and $\mathrm{e}^{2 \gamma l}$.

We can now solve equation 26 for the elements of $T_{B}$ : Taking ratios of the elements of $T_{B}$ we can show that

$$
\begin{gathered}
\frac{t_{21 B}}{t_{22 B}}=\frac{t_{21 T}-t_{11 T} \frac{t_{21 A}}{t_{11 A}}}{t_{22 T}-t_{12 T} \frac{t_{21 A}}{t_{11 A}}} \text { and } \\
\frac{t_{12 B}}{t_{11 B}}=\frac{t_{12 T}-t_{22 T} \frac{t_{12 A}}{t_{22 A}}}{t_{11 T}-t_{21 T} \frac{t_{12 A}}{t_{22 A}}} .
\end{gathered}
$$

Both of these quantities are therefore solved in terms of $\left(\frac{t_{11 A}}{t_{21 A}}\right),\left(\frac{t_{12 A}}{t_{22 A}}\right)$ and the measured quantities of $T_{T}$. Additionally, we can solve for

$$
\left(\frac{t_{11 B}}{t_{22 B}}\right)\left(\frac{t_{11 A}}{t_{22 A}}\right)=\frac{t_{11 T}-t_{21 T} \frac{t_{12 A}}{t_{22 A}}}{t_{22 T}-t_{12 T} \frac{t_{21 A}}{t_{11 A}}}
$$

which is known except for the quantity $\left(\frac{t_{11 A}}{t_{22 A}}\right)$.

We now know five parameters, $\left(\frac{t_{11 A}}{t_{21 A}}\right),\left(\frac{t_{12 A}}{t_{22 A}}\right),\left(\frac{t_{12 B}}{t_{11 B}}\right),\left(\frac{t_{21 B}}{t_{22 B}}\right)$ and $\mathrm{e}^{2 \gamma l}$. However, we have yet to make use of the reflection (short) measurements. The reflection coefficient measured at port $1, S_{11 R}$, can be written as a function of the reflective termination and the S-parameters of error-box A

$$
S_{11 R}=S_{11 A}+\frac{S_{12 A} S_{21 A} \Gamma_{L}}{1-S_{22 A} \Gamma_{L}}
$$

In terms of the T-matrix elements, this becomes

$$
S_{11 R}=\frac{\frac{t_{12 A}}{t_{22 A}}+\frac{t_{11 A}}{t_{22 A}} \Gamma_{L}}{1+\frac{t_{21 A}}{t_{22 A}} \Gamma_{L}}
$$


Solving equation 43 for $\left(\frac{t_{114}}{t_{22 A}}\right)$ gives

$$
\frac{t_{11 A}}{t_{22 A}}=\frac{S_{11 R}-\frac{t_{12 A}}{t_{22 A}}}{\Gamma_{L}\left(1-S_{11 R} \frac{t_{21 A}}{t_{11 A}}\right)} .
$$

Similarly, we can write for the reflection load at the B error box

$$
S_{22 R}=S_{22 B}+\frac{S_{12 B} S_{21 B} \Gamma_{L}}{1-S_{11 B} \Gamma_{L}}
$$

In terms of the T-matrix elements, we get

$$
S_{22 R}=\frac{-\frac{t_{21 B}}{t_{22 B}}+\frac{t_{11 B}}{t_{22 B}} \Gamma_{L}}{1-\frac{t_{12 B}}{t_{22 B}} \Gamma_{L}}
$$

Solving for $\left(\frac{t_{11 B}}{t_{22 B}}\right)$ gives

$$
\frac{t_{11 B}}{t_{22 B}}=\frac{S_{22 R}+\frac{t_{21 B}}{t_{22 B}}}{\Gamma_{L}\left(1+S_{22 R} \frac{t_{12 B}}{t_{11 B}}\right)} .
$$

We can now eliminate the unknown reflection coefficient, $\Gamma_{L}$ from equation 44 and equation 47 to get

$$
\left(\frac{t_{11 A}}{t_{22 A}}\right)\left(\frac{t_{22 B}}{t_{11 B}}\right)=\frac{\left(S_{11 R}-\frac{t_{12 A}}{t_{22 A}}\right)\left(1+S_{22 R} \frac{t_{12 B}}{t_{11 B}}\right)}{\left(1-S_{11 R} \frac{t_{21 A}}{t_{11 A}}\right)\left(S_{22 R}+\frac{t_{21 B}}{t_{22 B}}\right)}
$$

We can now combine equation 41 and equation 48 to get

$$
\frac{t_{11 A}}{t_{22 A}}= \pm \sqrt{\frac{\left(S_{11 R}-\frac{t_{12 A}}{t_{22 A}}\right)\left(1+S_{22 R} \frac{t_{12 B}}{t_{11 B}}\right)\left(t_{11 T}-t_{21 T} \frac{t_{12 A}}{t_{22 A}}\right)}{\left(1-S_{11 R} \frac{t_{21 A}}{t_{11 A}}\right)\left(S_{22 R}+\frac{t_{21 B}}{t_{22 B}}\right)\left(t_{22 T}-t_{12 T} \frac{t_{21 A}}{t_{11 A}}\right)}}
$$

and

$$
\frac{t_{11 B}}{t_{22 B}}=\frac{t_{11 T}-t_{21} \frac{t_{12 A}}{t_{22 A}}}{\left(\frac{t_{11 A}}{t_{22 A}}\right)\left(t_{22 T}-t_{12 T}\left(\frac{t_{21 A}}{t_{11 A}}\right)\right)} .
$$

The sign of equation 49 is still unknown. A nominal value for $\Gamma_{L}$ should be known, permitting the evaluation of the sign of equation 49 using equation 44 .

The TRL calibration is now complete and the scattering parameters of error box A are given from equations 30 through 33 as follows

$$
\begin{gathered}
S_{11 A}=\frac{t_{12 A}}{t_{22 A}} \\
S_{22 A}=-\frac{t_{21 A}}{t_{22 A}}=-\left(\frac{t_{21 A}}{t_{11 A}}\right)\left(\frac{t_{11 A}}{t_{22 A}}\right),
\end{gathered}
$$




$$
\begin{aligned}
S_{12 A} S_{21 A} & =\frac{1}{t_{22 A}^{2}}\left(t_{11 A} t_{22 A}-t_{12 A} t_{21 A}\right) \\
& =\frac{t_{11 A}}{t_{22 A}}\left\{1-\left(\frac{t_{12 A}}{t_{22 A}}\right)\left(\frac{t_{21 A}}{t_{11 A}}\right)\right\} .
\end{aligned}
$$

The parameters for error box B can be solved for in a similar manner or in a more convenient manner by using equation 26 giving

$$
\begin{gathered}
S_{11 B}=\frac{t_{12 T}-t_{22 T}\left(\frac{t_{21 A}}{t_{11 A}}\right)\left(\frac{t_{11 A}}{t_{22 A}}\right)}{t_{22 T}\left(\frac{t_{11 A}}{t_{22 A}}\right)-t_{12 T}\left(\frac{t_{12 A}}{t_{22 A}}\right)}, \\
S_{22 B}=\frac{t_{21 T}\left(\frac{t_{11 A}}{t_{22 A}}\right)-t_{11 T}\left(\frac{t_{12 A}}{t_{22 A}}\right)}{t_{22 T}\left(\frac{t_{11 A}}{t_{22 A}}\right)-t_{12 T}\left(\frac{t_{12 A}}{t_{22 A}}\right)}, \\
S_{12 B} S_{21 B}=\frac{t_{11 B}}{t_{22 B}}+S_{11 B} S_{22 B} \\
=\frac{t_{11 T}-t_{21 T}\left(\frac{t_{21 A}}{t_{11 A}}\right)\left(\frac{t_{11 A}}{t_{22 A}}\right)}{t_{22 T}\left(\frac{t_{11 A}}{t_{22 A}}\right)-t_{12 T}\left(\frac{t_{12 A}}{t_{22 A}}\right)}+S_{11 B} S_{22 B} .
\end{gathered}
$$

This completes the TRL solutions for error boxes A and B. It is quite easy to de-embed a measured S-matrix given this information.

As we can see, the TRL solution solves only for the error box product, $S_{12} S_{21}$. It is impossible, on physical grounds, to separate these two S-parameters. However, various assumptions can be made. First, we can note that the determinants of the measured Tmatrices have the following property

$$
\operatorname{DET}\left(T_{T}\right)=\operatorname{DET}\left(T_{D}\right)=\left(\frac{S_{12 A}}{S_{21 A}}\right)\left(\frac{S_{12 B}}{S_{21 B}}\right) .
$$

We can then hypothesize that the two error boxes share equally in the apparent nonreciprocity due to system error. This gives

$$
\operatorname{DET}\left(T_{A}\right)=\operatorname{DET}\left(T_{B}\right)
$$

Combining equation 57 with equation 58 results in the following relationship between the off-diagonal error box components

$$
\frac{S_{12 A}}{S_{21 A}}=\frac{S_{12 B}}{S_{21 B}}= \pm \sqrt{\frac{S_{12 T}}{S_{21 T}}} .
$$

This gives

$$
S_{12 A}= \pm \sqrt{ \pm S_{12 A} S_{21 A} \sqrt{\frac{S_{12 T}}{S_{21 T}}}}
$$


and

$$
S_{21 A}=\frac{S_{12 A} S_{21 A}}{S_{12 A}} .
$$

The product $S_{12 A} S_{21 A}$ is known from equation 53. Similar equations can be written for the $B$ error box off-diagonal components.

We must now assign the correct root in equation 37 to the correct ratio such that the solution is physically realizable. Choosing the roots correctly implies that the correct electrical length, $\gamma l$ is used. This may be done done by specifying that $\left|\mathrm{e}^{2 \gamma l}\right|<1$. By substituting the solutions for $\left(\frac{t_{11 A}}{t_{21 A}}\right)$ and $\left(\frac{t_{12 A} A}{t_{22 A}}\right)$ into equation 38 we get

$$
\mathrm{e}^{2 \gamma l}=\frac{t_{11 D T}+t_{22 D T} \pm \sqrt{\left(t_{11 D T}-t_{22 D T}\right)^{2}+4 t_{12 D T} t_{21 D T}}}{t_{11 D T}+t_{22 D T} \mp \sqrt{\left(t_{11 D T}-t_{22 D T}\right)^{2}+4 t_{12 D T} t_{21 D T}}}
$$

where the + sign in the numerator corresponds to setting the positive root of equation 38 equal to $\left(\frac{t_{12 A}}{t_{22 A}}\right)$. Thus, the magnitude of equation 62 may be used as a flag function to determine the correct root choice. Many times $\left|\mathrm{e}^{2 \gamma l}\right|$ is very close to one in value so this may not be the best test. Alternatively, if the electrical length of the calibration line is known with a fair degree of accuracy, the imaginary part of $\mathrm{e}^{2 \gamma l}$ may be used as a flag function. This is very accurate if a short spacer is used for the through measurement since the electrical

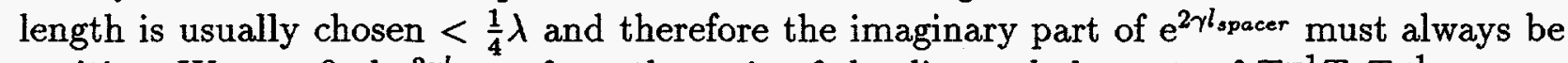
positive. We can find $\mathrm{e}^{2 \gamma l_{\text {spacer }}}$ from the ratio of the diagonal elements of $T_{A}^{-1} T_{T} T_{B}^{-1}$. 


\title{
Appendix B
}

\section{The De-Embedding Equations}

Once the components of the error matrices $\mathrm{A}$ and $\mathrm{B}$ have been determined, we can now de-embed a measured S-matrix and calculate the true S-matrix for the device under test. Expressed in terms of T-matrices the procedure is trivial and can be written as follows:

$$
T_{D U T}=T_{A}^{-1} T_{M} T_{B}^{-1}
$$

where $T_{D U T}$ is the true T-matrix and $T_{M}$ is the measured T-matrix for the device. Written explicitly in terms of the individual components of the S-matrices, we have [3]

$$
\begin{aligned}
S_{11} & =\frac{S_{11 B}\left[S_{11 A} S_{22 M}-\operatorname{DET}\left(S_{M}\right)\right]+\left(S_{11 M}-S_{11 A}\right) \operatorname{DET}\left(S_{B}\right)}{S_{11 B}\left[S_{22 M} \operatorname{DET}\left(S_{A}\right)-S_{22 A} \operatorname{DET}\left(S_{M}\right)\right]+\left[S_{11 M} S_{22 A}-\operatorname{DET}\left(S_{A}\right)\right] \operatorname{DET}\left(S_{B}\right)}, \\
S_{12} & =\frac{-S_{12 M} S_{21 A} S_{21 B}}{S_{11 B}\left[S_{22 M} \operatorname{DET}\left(S_{A}\right)-S_{22 A} \operatorname{DET}\left(S_{M}\right)\right]+\left[S_{11 M} S_{22 A}-\operatorname{DET}\left(S_{A}\right)\right] \operatorname{DET}\left(S_{B}\right)}, \\
S_{21} & =\frac{-S_{21 M} S_{12 A} S_{12 B}}{S_{11 B}\left[S_{22 M} \operatorname{DET}\left(S_{A}\right)-S_{22 A} \operatorname{DET}\left(S_{M}\right)\right]+\left[S_{11 M} S_{22 A}-\operatorname{DET}\left(S_{A}\right)\right] \operatorname{DET}\left(S_{B}\right)}, \\
S_{22}= & \frac{S_{22 B}\left[S_{22 A} S_{11 M}-\operatorname{DET}\left(S_{A}\right)\right]+S_{22 M} \operatorname{DET}\left(S_{A}\right)-S_{22 A} \operatorname{DET}\left(S_{M}\right)}{S_{11 B}\left[S_{22 M} \operatorname{DET}\left(S_{A}\right)-S_{22 A} \operatorname{DET}\left(S_{M}\right)\right]+\left[S_{11 M} S_{22 A}-\operatorname{DET}\left(S_{A}\right)\right] \operatorname{DET}\left(S_{B}\right)},
\end{aligned}
$$

where $\operatorname{DET}\left(S_{M}\right), \operatorname{DET}\left(S_{A}\right)$, and $\operatorname{DET}\left(S_{B}\right)$ are the determinants of the S-matrices for the measured device and the $\mathrm{A}$ and $\mathrm{B}$ error boxes, respectively. If the leakage terms have been determined, they may be easily added to the above equations by substituting $\left(S_{12 M}-S_{12 L}\right)$ for $S_{12 M}$ and $\left(S_{21 M}-S_{21 L}\right)$ for $S_{21 M}$.

\section{DISCLAIMER}

\begin{abstract}
This report was prepared as an account of work sponsored by an agency of the United States Government. Neither the United States Government nor any agency thereof, nor any of their employees, makes any warranty, express or implied, or assumes any legal liability or responsibility for the accuracy, completeness, or usefulness of any information, apparatus, product, or process disclosed, or represents that its use would not infringe privately owned rights. Reference herein to any specific commercial product, process, or service by trade name, trademark, manufacturer, or otherwise does not necessarily constitute or imply its endorsement, recommendation, or favoring by the United States Government or any agency thereof. The views and opinions of authors expressed herein do not necessarily state or reflect those of the United States Government or any agency thereof.
\end{abstract}

